\title{
HPLC-MS/MS method applied to an untargeted metabolomics approach for the diagnosis of "olive quick decline syndrome"
}

\author{
Sabrina Di Masi ${ }^{1}$ - Giuseppe E. De Benedetto ${ }^{2,7} \cdot$ Cosimino Malitesta $^{1,7} \cdot$ Maria Saponari $^{3} \cdot$ Cinzia Citti $^{4,5}$. \\ Giuseppe Cannazza $^{4,5} \cdot$ Giuseppe Ciccarella ${ }^{5,6,7}$
}

Received: 18 December 2020 / Revised: 9 February 2021 / Accepted: 5 March 2021 / Published online: 25 March 2021

(C) The Author(s) 2021

\begin{abstract}
Olive quick decline syndrome (OQDS) is a disorder associated with bacterial infections caused by Xylella fastidiosa subsp. pauca ST53 in olive trees. Metabolic profile changes occurring in infected olive trees are still poorly investigated, but have the potential to unravel reliable biomarkers to be exploited for early diagnosis of infections. In this study, an untargeted metabolomic method using high-performance liquid chromatography coupled to quadrupole-time-of-flight high-resolution mass spectrometry (HPLCESI-Q-TOF-MS) was used to detect differences in samples (leaves) from healthy (Ctrl) and infected (Xf) olive trees. Both unsupervised and supervised data analysis clearly differentiated the groups. Different metabolites have been identified as potential specific biomarkers, and their characterization strongly suggests that metabolism of flavonoids and long-chain fatty acids is perturbed in $\mathrm{Xf}$ samples. In particular, a decrease in the defence capabilities of the host after $X f$ infection is proposed because of a significant dysregulation of some metabolites belonging to flavonoid family. Moreover, oleic acid is confirmed as a putative diffusible signal factor (DSF). This study provides new insights into the host-pathogen interactions and confirms LCHRMS-based metabolomics as a powerful approach for disease-associated biomarkers discovery in plants.
\end{abstract}

Keywords Olive quick decline syndrome $\cdot$ Liquid chromatography $\cdot$ High-resolution mass spectrometry $\cdot$ Metabolomics

\section{Introduction}

Since 2010, olive trees of Salento (southeastern Italy) have been collapsing due to a severe disease called "olive quick decline syndrome" (OQDS) [1]. The disease has been associated with Xylella fastidiosa (Xf) infection, a Gramnegative pathogen colonizing xylem vessels, thus impairing water uptake. This bacterium is the well-known causal agent of other economically important diseases, i.e.

Published in the topical collection celebrating ABCs 20th Anniversary.

Cosimino Malitesta

cosimino.malitesta@unisalento.it

Giuseppe Ciccarella

giuseppe.ciccarella@unisalento.it

1 Laboratorio di Chimica Analitica, Dipartimento di Scienze e Tecnologie Biologiche ed Ambientali, Università del Salento, Via Monteroni, 73100 Lecce, Italy

2 Laboratorio di Spettrometria di Massa Analitica e Isotopica, Dipartimento di Beni Culturali, Università del Salento, Via Monteroni, 73100 Lecce, Italy
Pierce's disease [2] in grapes and leaf scorch of almond and other landscape and ornamental species. In the case of olive, the widespread occurrence of sapwood fungal infections (such as Phaeoacremonium and Phaeomoniella, Pleumostomophora and Neofusicoccum), probably exacerbates the effect of Xylella-infections [3]. However, the pathogenic role of $X$. fastidiosa subsp. pauca ST53 in the aetiology of OQDS has been clearly demonstrated by artificial inoculations on susceptible olive cultivars $[4,5]$.
3 Istituto per la Protezione Sostenibile delle Piante, CNR - IPSP, Consiglio Nazionale delle Ricerche, Via Amendola 165/A, 70126 Bari, Italy

4 Dipartimento di Scienze della Vita, Università di Modena e Reggio Emilia, Via Campi 103, 41125 Modena, Italy

5 Istituto di Nanotecnologia - CNR NANOTEC, Consiglio Nazionale delle Ricerche, Via Monteroni, 73100 Lecce, Italy

6 Dipartimento di Scienze e Tecnologie Biologiche ed Ambientali, Università del Salento, Via Monteroni, 73100 Lecce, Italy

7 UdR INSTM, Università del Salento, Lecce, Italy 
This study also unraveled the long incubation period of the infections in olives. In fact, the onset of symptoms occurred more than 1 year after the bacterial inoculation [6]. In this scenario, the possibility of making an early diagnosis of the infections is of utmost importance. To date, it is possible to detect the presence of $X f$ in olive trees by serological and molecular methods [7]. However, the long latency period of the infections and the irregular distribution of the bacterium in the trees (in particular, in century-old trees of relevant size) prompted the exploration of alternative tools to unravel the presence of the infections by detecting bacterial compounds involved in the infection process, including those related to the host response.

Metabolomics emerged within the omics technologies as a valuable aid to understand complex molecular complex in biological systems [8] and to provide a useful evaluation of the cellular state at a molecular level. In fact, metabolites can be observed as the end-products of gene expression and can represent a "fingerprint" of a cell or tissue. Moreover, they generally change within either host or pathogen in response to their specific interactions. In a biological system, the comprehensive analysis of all metabolites is a difficult target, not yet reached for any system. Indeed, the past few years have seen great advances in high-throughput metabolomics, after integration with the other "omics" through bioinformatics [8, 9], making significant progress into understanding different biological processes.

In the recent efforts to comprehend and face OQDS, different analytical procedures have been widely employed to achieve adequate metabolite coverage. A GC-MS method [10] has been adopted to highlight differences in the composition of volatile organic compounds (VOC) between healthy and infected olive tree samples. In a recent work, an LC-MS untargeted lipidomic analysis of infected Olea europaea samples revealed a shortlist of molecules that modulate biofilm phases in $X$. fastidiosa subsp. pauca [11].

The basic philosophy of an untargeted metabolomics approach is to detect as many metabolites as possible to maximize the likelihood of identifying compounds that are dysregulated in a biological condition. In this research work, using an untargeted metabolomics approach, we attempted to identify metabolites produced by the pathogen or metabolites that represent the biochemical response of the host to the infection. An efficient analytical method based on a liquid chromatography separation coupled to high-resolution mass spectrometry (LC-HRMS) permitted us to unravel the main differences between healthy and Xylella-infected trees. Feature extraction and two-group analysis was performed by the XCMS online software. Significant features were used to distinguish samples taken from healthy $(\mathrm{Ctrl})$ and infected $(\mathrm{Xf})$ olive trees and to perform partial least square discriminant analysis. To validate our experimental model, cross-model validation and permutation tests were run. Online databases also allowed for the putative identification of some of the metabolites involved in OQDS. The relevant results are herein discussed.

\section{Materials and methods}

\section{Sampling}

Field samples were collected from olive trees in two different areas of the Salento Peninsula. Ten leaves collected from different brushes of each sample tree were immediately shockfrozen with liquid nitrogen to block all metabolic processes and transferred to the laboratory for extraction. All sampled plants were without OQDS symptoms at different distances from the infected (desiccated) ones. Table S1 (see Supplementary Information, ESM) lists the sample label, the cultivar and healthy/infected state based on real-time PCR assays carried out as already reported [9].

\section{Extraction protocol and sample preparation}

The extraction procedure is a critical step prior to metabolomic experiments. First, the leaves were immersed in liquid nitrogen, then manually ground with a pestle and a mortar (pre-cooled and filled with liquid nitrogen). For the extraction procedure, ethyl acetate was chosen, which was reported to be the best extraction solvent in terms of number of metabolites with large chemical and structural diversity detected by MS [12-14]. Therefore, $300 \mathrm{mg}$ of fine powder was extracted with $1.2 \mathrm{~mL}$ of ethyl acetate in $1.5 \mathrm{~mL}$ Eppendorf tubes, sonicated for $15 \mathrm{~min}$, and centrifuged at $12,000 \mathrm{rpm}$ for $20 \mathrm{~min}$. $500 \mu \mathrm{L}$ of the supernatant was transferred into a new Eppendorf tube and the solvent was evaporated under a stream of nitrogen. The addition of $1 \mathrm{~mL}$ of water/acetonitrile 50:50 (v/v) to the dry extract was followed by sonication (15 $\mathrm{min}$ ) and centrifugation (12,000 rpm, $20 \mathrm{~min})$. The supernatants were transferred into HPLC vials and analyzed by LC-MS.

\section{HPLC-ESI-QTOF analysis}

Analyses were performed on an Agilent 1200 series liquid chromatograph (LC) equipped with a solvent vacuum degasser, a binary pump, a thermostated autosampler, a thermostated column compartment and a photodiode array detector (DAD) and interfaced to an Agilent 6540 Q-TOFMS equipped with an ESI source. A general-purpose C18 column (Poroshell 120 SB-C18, $2.1 \times 100 \mathrm{~mm}, 2.7 \mu \mathrm{m}$, Agilent, Milano, Italy) was employed and metabolites were separated using a mobile phase composed of $0.1 \%$ formic acid in water (A) and $0.1 \%$ formic acid in acetonitrile (B). The gradient elution was set as follows: linear gradient from 5 to $95 \% \mathrm{~B}$ in $45 \mathrm{~min}, 95 \% \mathrm{~B}$ held for $10 \mathrm{~min}$, linear gradient from 95 to $5 \% \mathrm{~B}$ in $5 \mathrm{~min}$ and $5 \% \mathrm{~B}$ held for $5 \mathrm{~min}$ to equilibrate the 
column at the initial conditions. The flow rate was $300 \mu \mathrm{L} /$ $\min$. The run time was $65 \mathrm{~min}$. The column temperature was set at $25{ }^{\circ} \mathrm{C}$. The sample injection volume was $5 \mu \mathrm{L}$. DAD acquisitions were carried out in the range of $190-600 \mathrm{~nm}$. The mass spectrometer was operated in full-scan mode in the $\mathrm{m} / \mathrm{z}$ range $50-1700$ with a scan rate of $1.42 \mathrm{spectra} / \mathrm{s}$, in both positive and negative ionization modes with the following experimental parameters: the capillary voltage was $3.5 \mathrm{kV}$, the nebulizer $\left(\mathrm{N}_{2}\right)$ pressure was $30 \mathrm{psi}$, the drying gas temperature was $350{ }^{\circ} \mathrm{C}$, the drying gas flow was $12 \mathrm{~L} / \mathrm{min}$ and the skimmer voltage was $40 \mathrm{~V}$. Internal calibration was achieved by enabling constant check of reference masses in the calibration mix solution introduced to the ion source via the dual ESI port. To identify the molecular structure of significant ions, all samples were also acquired in both positive and negative ionization modes using the auto MS/MS function, nitrogen as the collision gas, and a collision energy of $20 \mathrm{eV}$, under the same experimental conditions described above. All the measurements were run and recorded with Agilent Mass Hunter software (Rev. B.01.04).

\section{Data processing}

LC/MS data were first processed using XCMS Online software (https://xcmsonline.scripps.edu) [15]. Raw LC-MS data files were converted into $m z M L$ files and uploaded to the online platform in two datasets, containing $17 \mathrm{Ctrl}$ samples and $10 \mathrm{Xf}$ samples. Additional data from samples of different cultivars (18-22 Ctrl samples) were treated with the same procedure. The following parameters were used to process either positive or negative ion mode data in different pairwise jobs: feature detection with the centWave algorithm $(\Delta m / z=$ $15 \mathrm{ppm}$, minimum and maximum peak width 10 and $60 \mathrm{~s}$, respectively; $\mathrm{S} / \mathrm{N}$ threshold $=6$ ); retention time correction with the usual obiwarp settings (profStep $=0.5$ ); chromatogram alignment with $\mathrm{mzwid}=0.015$, $\operatorname{minfrac}=0.5$, and $\mathrm{bw}=$ 5 ; parameters for annotation, including ppm error $=5$ and $\mathrm{m} / \mathrm{z}$ absolute error $=0.015$. The results of these pairwise calculations are presented in a table in which detailed information about each single metabolite $(\mathrm{m} / \mathrm{z}, \mathrm{p}$ value, fold-change, peak intensity, retention time, extracted ion chromatogram (EIC), mass spectrum and box plot) are reported. The identification of metabolites was achieved by MS/MS spectrum match with MS-FINDER, which was able to elucidate the formula and chemical structures from accurate mass precursor ions and MS/MS spectra [16]. The XCMS csv output file was imported in SIMCA (version 16). This software was used to perform principal component analysis (PCA), partial least square discriminant analysis (PLS-DA) and orthogonal projections to latent structures discriminant analysis (OPLS-DA) and to validate the models. Data were Pareto scaled before performing PCA, PLS-DA and OPLS-DA methods.

\section{Results}

\section{Data analysis}

The $m z M L$ data files were processed with XCMS Online software using pairwise analysis, which allows the comparison of two groups "control" and "disease", i.e. healthy and Xfinfected plants. The analyses were carried out by ESI-MS in either positive or negative ion mode. The feature detection carried out by XCMS Online with the centWave method permitted us to extract 26,604 and 9598 features for + and - ion mode data, respectively, whereas the parametric independent (unpaired) two-group tests (Welch $t$ test) permitted us to determine the metabolite features whose levels are significantly different. Figure 1 gives the actual representation of global metabolomic data in ESI-MS (both + and - ion mode): the relevant cloud plots (that can be exported directly from XCMS Online) show those features exceeding both $p$ value and FC thresholds, set at 0.01 and 1.5 , respectively, distributed along the chromatogram in the upper or lower panel according being up or downregulated by the $\mathrm{Xf}$ infection.

The number of features with a $p$ value $<0.01$ and fold change $>1.5$ were 14.437 in positive ion mode and 6,236 features in negative ion mode. These findings confirm the greatest sensitivity of ESI-MS (+ ion mode) in global metabolomic analysis.

The patterns in the data were searched by PCA, an unsupervised multivariate method useful to examine whether the detected features are able to group samples. This method is "unsupervised" because it is carried out without data labeling with class membership. It is also a strong method to dimensionally reduce a data set containing thousands of metabolites, calculating the few combinations best explaining original data variance. Figure 2 shows the PCA score plots, showing its capability to separate healthy and Xf-infected olive tree samples along PC1 and PC2. The first two PCs explained 67.6\% and $7.25 \%$ of the total variance in ESI (+ ion mode, panel A) and the $62.5 \%$ and $7.7 \%$ in ESI (- ion mode, panel B).

These results suggested that the PCA represents an effective tool to distinguish Ctrl (healthy) and Xf (infected) olive trees. Actually, notwithstanding there are two different cultivars, the trend is unique, and no outlier has been identified. Filtering out those features that did not change significantly between the two groups, i.e. using the same features shown in the cloud plot of Fig. 1, the effectiveness of PCA in distinguishing the two groups was confirmed. As a result, a valid classification of these metabolomic data could be obtained [17] and PLS-DA and OPLS-DA were used to further investigate the discrimination ability of these significant metabolites. Both methods are among the most often used supervised method in metabolomics and maximize the covariance between experimental data and dummy-dependent y-variablecontaining group labels. Moreover, OPLS-DA permits the 
a Cloud Plot 14437 features with $p$-value $\leq 0.01$, fold change $\geq 1.5$

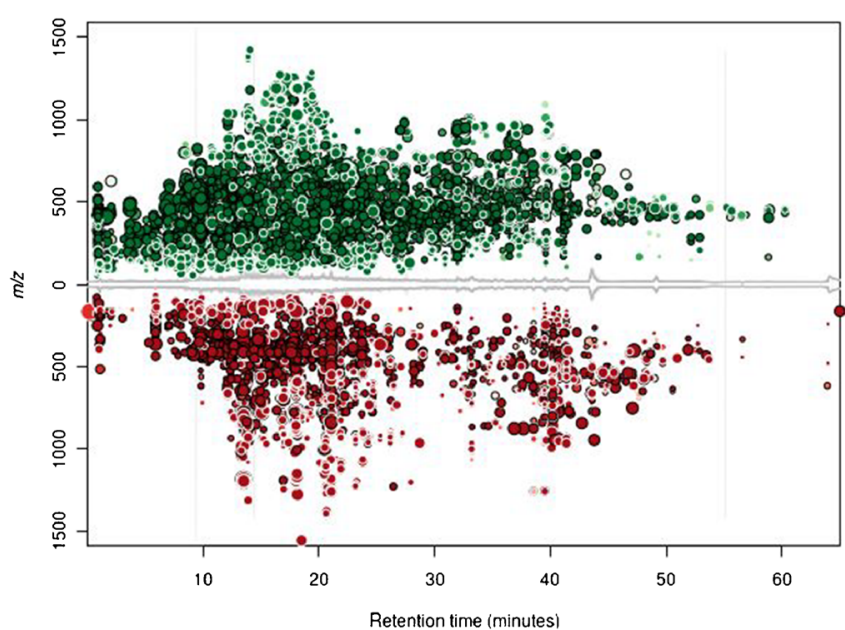

b Cloud Plot 6236 features with p-value $\leq 0.01$, fold change $\geq 1.5$

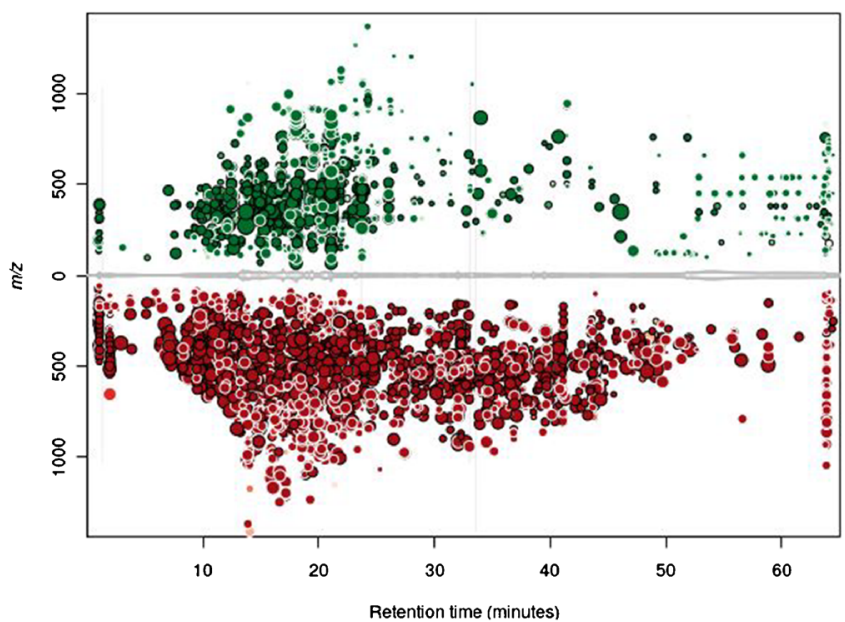

Fig. 1 XCMS Cloud plot representation of the dysregulated metabolite features for ESI-MS (a) positive ion mode and (b) negative ion mode: green bubbles represent the up-regulated features whereas the red ones represent the down-regulated features. Bubble diameter is proportional to fold change

separation of group-predictive and group-unrelated variance, calculating the further components orthogonal to the first. The model calibration was performed on about $80 \%$ of samples of each class, i.e. on 22 samples, whereas the training set consisted of the remaining ones as reported in Table 1.

The selection of latent variables and the cross validation of the model was performed using cancellation groups on the basis of Venetian blinds because the samples are ordered. Obviously, at this point, only the training set (WS or working set in SIMCA) is considered. Two different validation runs ( 3 and 4 cancellation groups) gave similar results in terms of error rate: in order to not overfit experimental data, 2 latent variables and 3 cancellation groups were selected to calculate the final models. Their performance is summarized in Table 2.

The reliability of the models can also be ascertained from the obtained PLS-DA (Fig. 3a and b) and OPLS-DA (Fig. 3c and d) score plots: all the samples, in both the training set and the test set, have been plotted in the figures and the modeling capabilities are evident, with a correct classification of all the test samples that have not been used in the model fitting (ESM Table S2). The model is also robust towards samples not belonging to Ogliarola and Cellina, which represent the cultivars that have been considered in the present work: 5 samples of different cultivars, all not affected by OQDS are correctly classified as healthy (see ESM, Fig. S2 and Tables S3-S6).
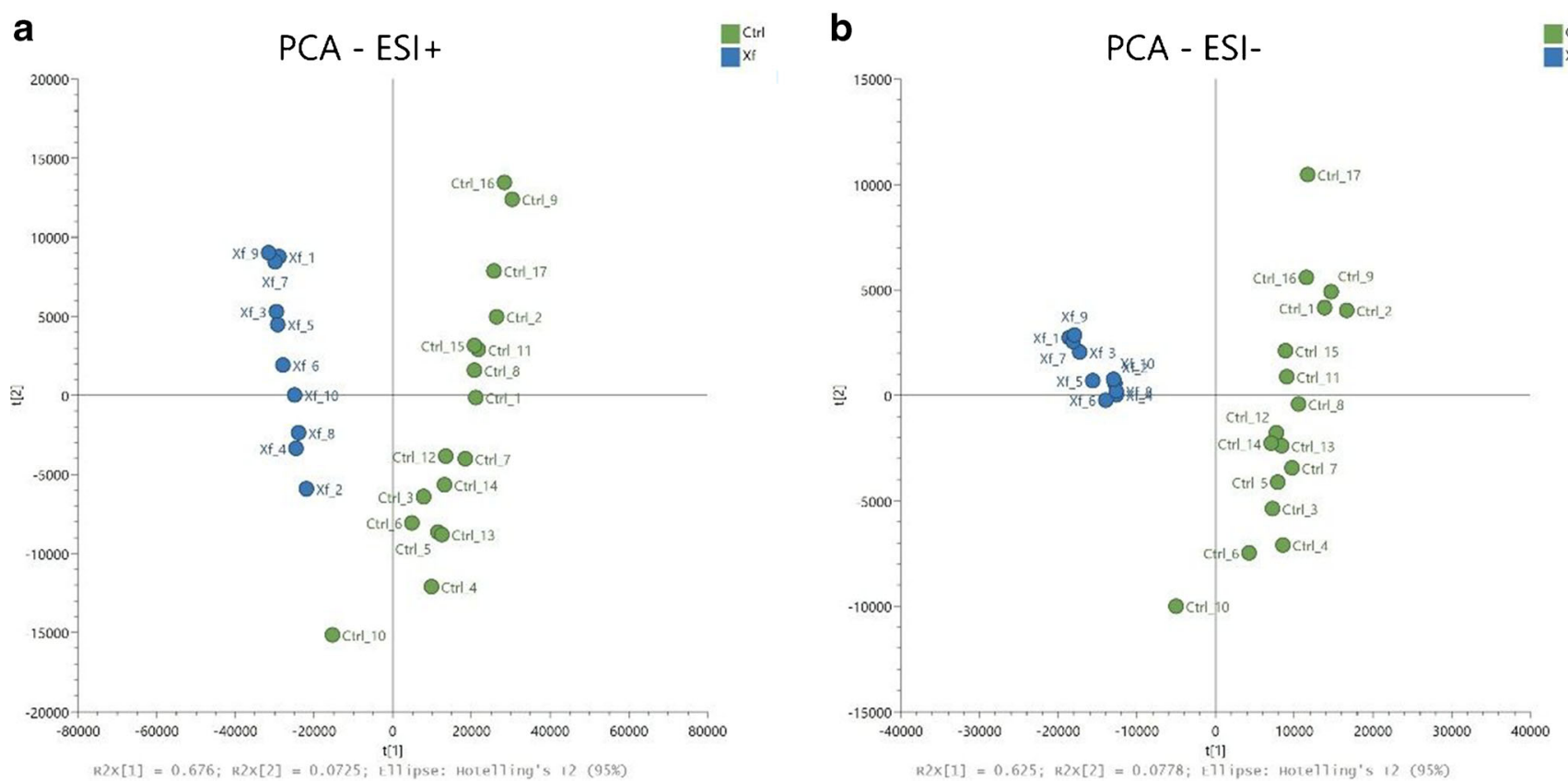

Fig. 2 Score plot of principal component analysis (PCA) on metabolomic data acquired in ESI-MS (a) positive ion mode and (b) negative ion mode 
Table 1 Sample partition in Ctrl (healthy olive trees) and Xf (infected ones) classes, as well as in training and test sets

\begin{tabular}{llll}
\hline & Ctrl (healthy olive trees) & Xf (infected olive trees) & Total \\
\hline Training set & 14 & 8 & 22 \\
Test set & 3 & 2 & 5 \\
Total & 17 & 10 & 27 \\
\hline
\end{tabular}

Feature annotation, i.e. the metabolite identification, is a key step to find those biomolecule markers of the infection that are potentially useful to develop a new analytical method based on a targeted metabolomic approach aiming at an early diagnosis of the infection. Among these thousands of features, only those exhibiting the most significant fold changes were searched in detail. Tentative metabolite identification was based on the in silico MINE database and in the PubChem compound database available in MS-FINDER, which also allows us to score and rank candidate structures. Tables 2 lists some of the significant metabolites with their retention time, accurate $\mathrm{m} / \mathrm{z}$ value, fragment ions, fold change, $p$ value, and ion mode. This software was also used to confirm annotation by comparing the experimental MS/MS spectra with the computer generated ones. The high-resolution MS/MS spectra of metabolites identified and discussed below are reported in Fig. S1 (see ESM).

As can be seen in Table 3, some flavone and flavonoid-Oglucosides were detected as significantly dysregulated. This result is not surprising because flavonoids are widely distributed and some of them are involved in response to microbial attack in different host-pathogen systems [18]. Changes in the levels of metabolites belonging to the class of fatty acids, both saturated and unsaturated, were also recorded in the chromatograms. In Fig. 4 the extracted ion chromatograms for Ctrl and Xf samples (ESI-MS, - ion mode) shows how palmitic acid and stearic acids are upregulated in Xf-infected samples. Oleic acid was also upregulated, with a 6-fold increase in the Xf samples.

\section{Discussion}

In the present work, we employed an untargeted metabolomics approach to identify the main changes in the metabolic profile of olive leaves affected by the "olive quick decline syndrome". Significant upregulated or downregulated metabolites detected by ESI-MS (either in + or - ion mode) and reported in Tab. 3 are discussed below.

Diosmin. The deprotonated molecule $[\mathrm{M}-\mathrm{H}]^{-}$was observed in the ESI-MS (- ion mode) at $\mathrm{m} / \mathrm{z} 607.1614$. Diosmin is a polyphenol with a flavonoid structure, showing high solubility in water, and its presence appears to be included in defence response activities by blocking protein synthesis in viruses [19]. In our samples, the diosmin was downregulated in Xf samples, which may be ascribed to the defence mechanisms being activated in infected plants.

Diosmetin 7-O-beta-D-glucopyranoside has been also identified in ESI-MS (- ion mode) as a downregulated metabolite in $X f$ samples (5.3-fold) at $\mathrm{m} / \mathrm{z} 461.0673$ and it is often cited as having antioxidant and antimicrobial effects [20].

Taxifolin (also known as dihydroquercetin), having a chemical formula of $\mathrm{C}_{15} \mathrm{H}_{12} \mathrm{O}_{7}$ was identified at $\mathrm{m} / \mathrm{z}$ 303.0485 (ESI-MS, - ion mode). The potential interest in taxifolin is mainly focused on its antioxidant properties. In our results, the amount of taxifolin was downregulated with a 6.7-fold change.

Ligstroside. Ligstroside is a glucoside secoiridoid involved in plant metabolism. It is a secondary plant metabolite having a chemical structure containing one phenol ring and a formula of $\mathrm{C}_{25} \mathrm{H}_{32} \mathrm{O}_{12}$ and was identified at $\mathrm{m} / \mathrm{z} 523.1752$ in ESI-MS (- ion mode). Ligstroside is generally present in olive trees' lipidic fraction and in extra-virgin olive oil (EVOO), and its antioxidant, anticarcinogenic, anti-inflammatory and immunomodulatory properties have been assessed [21]. In our study, this metabolite is downregulated, and it is discriminative for the infected leaves (1.9-fold change) in comparison with infected ones (Xf).

Kaempferol and Luteolin. These metabolites are significantly downregulated, with up to a 5.6-fold change measured

Table 2 PLS-DA and OPLS-DA models component description and predictive performances

\begin{tabular}{|c|c|c|c|c|c|c|c|c|}
\hline & Component & $\mathrm{R} 2 \mathrm{X}$ & R2X (cum) & Eigenvalue & $\mathrm{R} 2 \mathrm{Y}$ & R2Y(cum) & Q2 & Q2 (cum) \\
\hline \multirow[t]{2}{*}{ PLS-DA (ESI-MS data, positive ion mode) } & 1 & 0.629 & 0.629 & 13.8 & 0.859 & 0.859 & 0.844 & 0.844 \\
\hline & 2 & 0.0609 & 0.69 & 1.34 & 0.127 & 0.986 & 0.464 & 0.916 \\
\hline \multirow[t]{2}{*}{ PLS-DA (ESI-MS data, negative ion mode) } & 1 & 0.601 & 0.601 & 13.2 & 0.83 & 0.83 & 0.814 & 0.814 \\
\hline & 2 & 0.0593 & 0.66 & 1.31 & 0.145 & 0.975 & 0.526 & 0.912 \\
\hline \multirow[t]{2}{*}{ OPLS-DA (ESI-MS data, positive ion mode) } & P1 (Predictive) & 0.542 & 0.542 & 11.9 & 0.986 & 0.986 & 0.917 & 0.917 \\
\hline & O1 (Orthogonal in X) & 0.149 & 0.149 & 3.27 & & & & \\
\hline \multirow[t]{2}{*}{ OPLS-DA (ESI-MS data, negative ion mode) } & P1 (Predictive) & 0.504 & 0.504 & 11.1 & 0.975 & 0.975 & 0.874 & 0.874 \\
\hline & O1 (Orthogonal in X) & 0.156 & 0.156 & 3.44 & & & & \\
\hline
\end{tabular}


Fig. 3 Scatter plots for the predicted scores of the two components retained in the PLSDA and OPLS-DA models calculated for both ESI-MS ion modes: (a) PLS-DA, ESI-MS, + ion mode; (b) PLS-DA, ESI-MS, - ion mode; (c) OPLS-DA, ESIMS, + ion mode; and (d) OPLSDA, ESI-MS, - ion mode. Healthy (Ctrl) and Xf-infected samples (Xf) belonging to the training set are coloured in green and in red, respectively, whereas all the test samples are in blue. Variables were filtered according to $p$ value $<0.01$ and fold change $>1.5$ for both ESI modes a

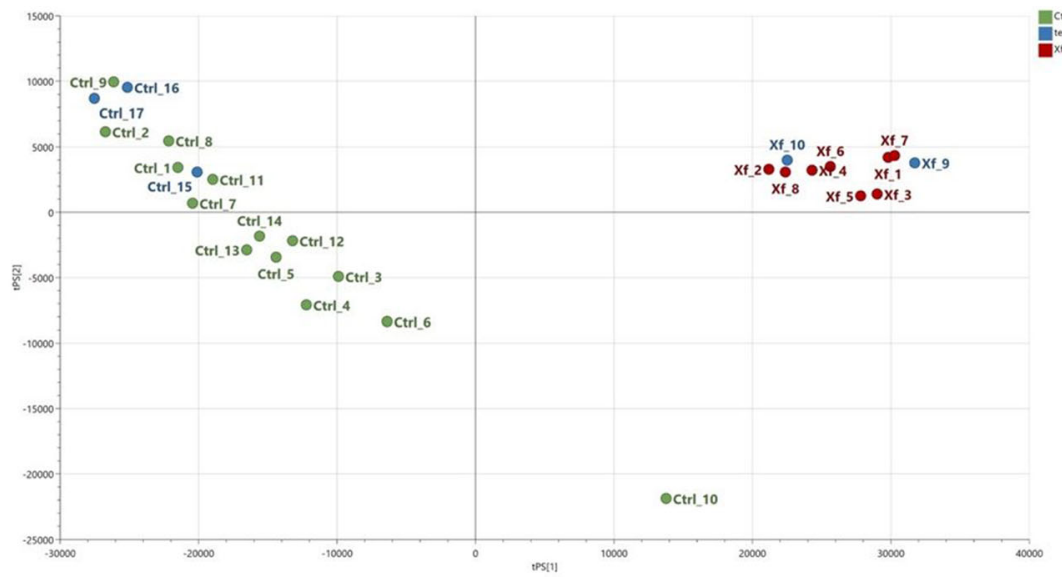

b

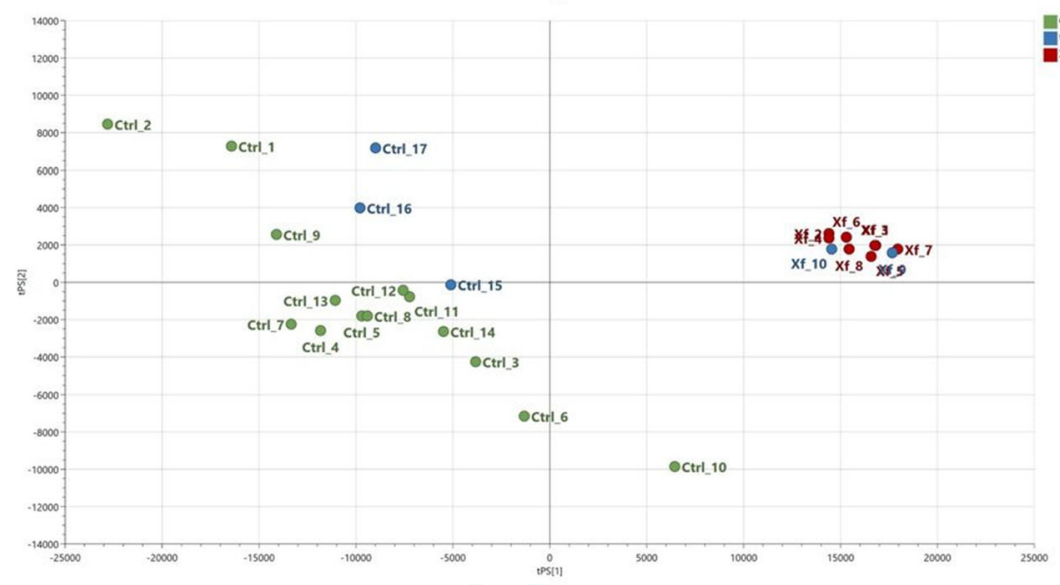

C

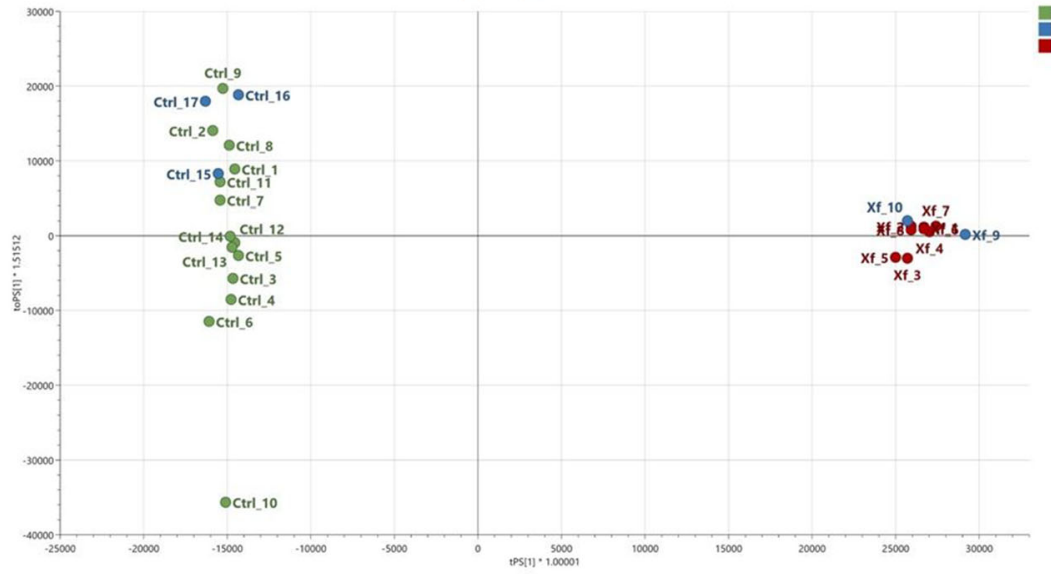

d

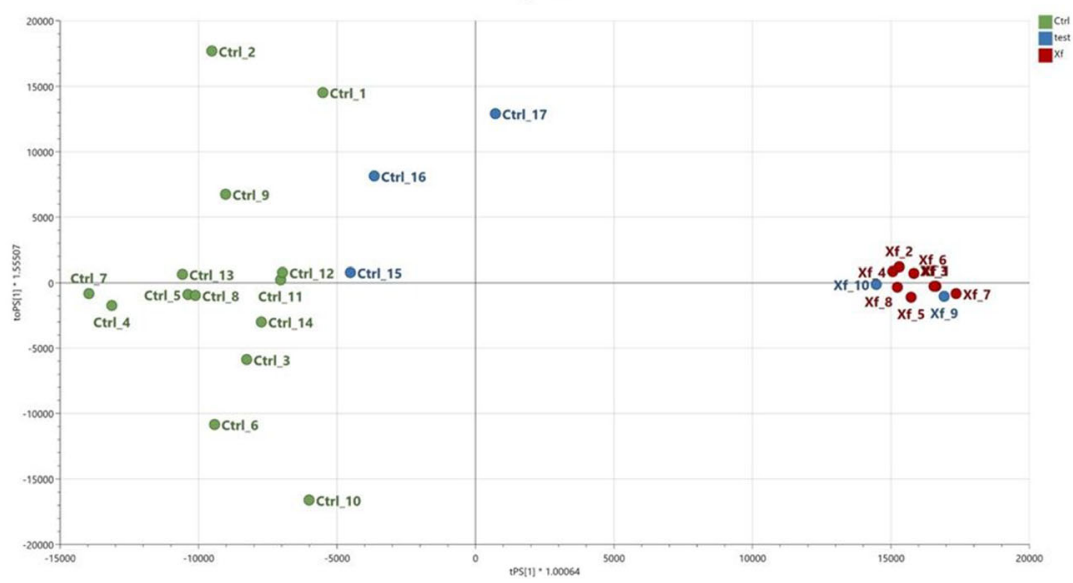


for kaempferol. Both kaempferol and luteolin have the same molecular formula and mass and were distinguished by their different MS/MS spectra (ESM Fig. S1). It has been widely reported that flavonoid content in plants is responsible for antimicrobial activity, especially towards Gram-negative bacteria [22].The results in the present study thus illuminate one of the possible effects of microbial attack, i.e. the demolition of the antioxidant potential of flavonoids, paving the way for rapid infection of the Xf-attacked plants.

Solavetivone has been identified at $\mathrm{m} / z 219.1653$ in ESIMS (+ ion mode). This is a stress compound with a sesquiterpenoid structure, and is thus implicated in disease resistance [23]. It is most likely produced by the plant as a result of post-infection stress. Given that it is expressed in major concentrations in Xf leaves (12.6-fold higher with respect to the controls), this molecule could represent a biological marker of infection.

Sinapic acid. The metabolite having a m/z 225.0747 ESIMS (+ ion mode) was identified as sinapic acid, which is constitutively expressed in the leaves of healthy trees. But, its concentration decreases (3.4 times) when the plant is in a state of stress.

Pyridoxine (Vitamin B6). Pyridoxine has been identified at $\mathrm{m} / \mathrm{z} 170.0804$ in ESI-MS (+ ion mode). In the context of selfdefence against oxidative stress, we found that pyridoxine, one of the six isoforms of vitamin B6, was expressed with concentrations 8.4 times higher by the infected trees,

Table 3 Significant metabolites identified in healthy (control) and Xf samples whose dysregulation between not-infected and infected plant samples is discussed in the text. For each recognized metabolite, along with electrospray mode, formula, exact mass, and retention time, the

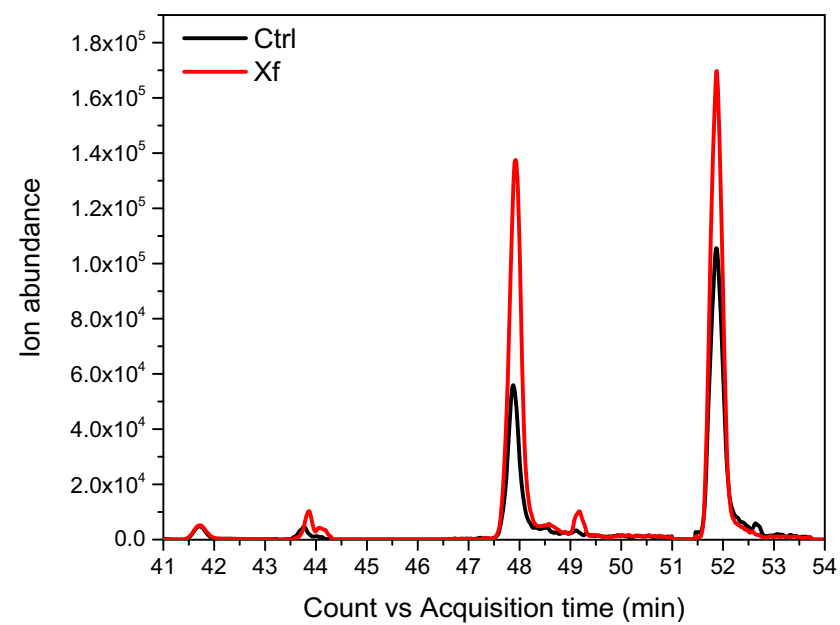

Fig. 4 Comparison of extracted ion chromatogram (EIC) of some fatty acid metabolites in ESI-MS (negative ion mode) between Ctrl and Xfinfected samples

indicating that the plant had implemented its defences against the infection. Moreover, it has been recently reported that vitamin B6 could play a key role as a signalling molecule to alert the plant of the need for ammonium, a nitrogen source necessary for the biosynthesis of vital compounds, such as amino acids and proteins [24].

Abscisic acid. The deprotonated plant hormone abscisic acid $[\mathrm{M}-\mathrm{H}]^{-}$was found at $m / z$ 263.1264 in ESI-MS (- ion mode). Low-energy CID-MS/MS analysis of this precursor

values explaining the differences are reported: fold change, $p$ value and dysregulation trend. These metabolites have been confirmed by MS/MS experiments (ESM Fig. S1)

\begin{tabular}{|c|c|c|c|c|c|c|c|}
\hline Metabolite & ESI-MS ion mode & Formula & $m / z$ & $\mathrm{RT}(\min )$ & Fold change & $\mathrm{p}$ value & Change trend \\
\hline Pyridoxine & + & $\mathrm{C}_{8} \mathrm{H}_{11} \mathrm{NO}_{3}$ & 170.0804 & 1.1 & 8.4 & $1.23 \mathrm{E}-06$ & UP \\
\hline$\beta$-Ionone & + & $\mathrm{C}_{13} \mathrm{H}_{20} \mathrm{O}$ & 193.1577 & 11.42 & 30.2 & $2.17 \mathrm{E}-08$ & UP \\
\hline Taxifolin & - & $\mathrm{C}_{15} \mathrm{H}_{12} \mathrm{O}_{7}$ & 303.0485 & 12.13 & 6.7 & $1.82 \mathrm{E}-06$ & DOWN \\
\hline Kaempferol & - & $\mathrm{C}_{15} \mathrm{H}_{10} \mathrm{O}_{6}$ & 285.0376 & 12.14 & 5.6 & $3.69 \mathrm{E}-06$ & DOWN \\
\hline Solavetivone & + & $\mathrm{C}_{15} \mathrm{H}_{22} \mathrm{O}$ & 219.1663 & 13.19 & 12.6 & $4.35 \mathrm{E}-12$ & UP \\
\hline Diosmin & - & $\mathrm{C}_{28} \mathrm{H}_{32} \mathrm{O}_{15}$ & 607.1614 & 13.88 & 2.0 & $1.11 \mathrm{E}-03$ & DOWN \\
\hline Diosmetin 7-O-beta-D-glucopyranoside & - & $\mathrm{C}_{22} \mathrm{H}_{22} \mathrm{O}_{11}$ & 461.0673 & 13.88 & 5.3 & $6.43 \mathrm{E}-10$ & DOWN \\
\hline Jasmonic acid & - & $\mathrm{C}_{12} \mathrm{H}_{18} \mathrm{O}_{3}$ & 209.1180 & 15.26 & 8.2 & $1.22 \mathrm{E}-07$ & UP \\
\hline (S)-Abscisic acid & - & $\mathrm{C}_{15} \mathrm{H}_{20} \mathrm{O}_{4}$ & 263.1264 & 15.67 & 3.3 & $9.05 \mathrm{E}-09$ & UP \\
\hline Ligstroside & - & $\mathrm{C}_{25} \mathrm{H}_{32} \mathrm{O}_{12}$ & 523.1752 & 16.09 & 1.9 & $3.96 \mathrm{E}-03$ & DOWN \\
\hline Luteolin & - & $\mathrm{C}_{15} \mathrm{H}_{10} \mathrm{O}_{6}$ & 285.0376 & 12.14 & 5.6 & $3.69 \mathrm{E}-07$ & DOWN \\
\hline Sinapic acid & + & $\mathrm{C}_{11} \mathrm{H}_{14} \mathrm{O}_{5}$ & 225.075 & 20.35 & 3.4 & $3.00 \mathrm{E}-04$ & DOWN \\
\hline Maslinic acid & - & $\mathrm{C}_{30} \mathrm{H}_{48} \mathrm{O}_{4}$ & 471.3472 & 30.50 & 3.3 & 2.72E-09 & UP \\
\hline 12-HETE & - & $\mathrm{C}_{20} \mathrm{H}_{32} \mathrm{O}_{3}$ & 319.2298 & 32.30 & 5.0 & $3.00 \mathrm{E}-05$ & DOWN \\
\hline Palmitic acid & - & $\mathrm{C}_{16} \mathrm{H}_{32} \mathrm{O}_{2}$ & 255.2330 & 36.52 & 10.5 & $3.00 \mathrm{E}-05$ & UP \\
\hline Heptadecanoic acid & - & $\mathrm{C}_{17} \mathrm{H}_{34} \mathrm{O}_{2}$ & 269.2489 & 36.86 & 7.9 & $4.05 \mathrm{E}-06$ & UP \\
\hline Oleic acid & - & $\mathrm{C}_{18} \mathrm{H}_{34} \mathrm{O}_{2}$ & 281.2504 & 37.76 & 6.0 & $5.00 \mathrm{E}-05$ & UP \\
\hline Stearic acid & - & $\mathrm{C}_{18} \mathrm{H}_{36} \mathrm{O}_{2}$ & 283.2670 & 51.87 & 1.6 & $6.00 \mathrm{E}-04$ & UP \\
\hline
\end{tabular}


ion was initiated by loss of carbon dioxide to afford the [M$\left.\mathrm{CO}_{2}\right]^{-}$product ion at $\mathrm{m} / z 220.97$ [25]. In our study, the abscisic acid was upregulated, with a 3.3 -fold change. Concurring with the regulation of stomatal aperture and adaptation to drought, low temperature and salinity, this compound clearly plays a role in the plant response to Xf infection [26].

Jasmonic acid at m/z 209.1180 was identified in ESI-MS (-ion mode) and it is upregulated in samples from infected plants. Considering that jasmonate regulates plant responses to different stresses such as drought [27], this endogenous rise in JA, as observed in the present work, can be seen as a host response to infection.

Ionone. Ionone is a volatile organic compound ( $\mathrm{VOC})$ whose concentration is about 30 times higher in infected samples, suggesting its implication in the defence-resistance process. As a VOC, indeed, it functions both as a defence factor and as a signalling molecule, [28] and it could have a key role in a range of interactions between plants (allelopathy) and between plants and non-plant organisms.

Fatty acids (FAs). It is noteworthy to mention that oleic acid belongs to a class of unsaturated fatty acids which have been proposed to be involved in Xf quorum sensing, a cell-tocell communication system $[5,13]$. These pathogen-derived lipid molecules, known as diffusible signal factors (DSF) [29] play a key role in Xf, as they modulate a wide set of biochemical processes. A complex mechanism of DSF regulation, based on population size, influences the expression of several $\mathrm{Xf}$ virulence traits, such as biofilm formation, adhesiveness and motility [30]. Moreover, the higher concentration of oleic acid in Xf samples is consistent with recent findings [11], which report a significant accumulation of this compound in Xf-infected olive samples. In light of this finding, we believe that selected FAs may also be considered as valuable markers of OQDS infection.

To summarize, an untargeted metabolomics approach was applied to olive leaf samples with the aim of understanding the main differences between healthy plants and plants with OQDS. An extraction procedure with ethyl acetate was confirmed to be an effective extraction method, permitting the detection of thousands of features. The results of multivariate analysis showed a perfect clustering of the two pools of samples (Ctrl and $\mathrm{Xf}$ ) based on two principal components (PC1 and PC2). The first allowed the separation of healthy from infected samples, and the second permitted distinction among different cultivars. Based on these data, PLS models were calculated and validated. A few molecules associated with features displaying significant changes between the two data sets could be identified: some are substances implicated in host response to infections, others are involved in Xf quorum sensing. A significant dysregulation of some metabolites belonging to the flavonoid family, evidencing a decrease in the host's defence capabilities after $X f$ infection, has been shown for the first time. Once these results are confirmed on a wider sample, they also may be considered as markers for an early diagnosis of OQDS. This would help in planning a strategy for an efficient therapy.

Supplementary Information The online version contains supplementary material available at https://doi.org/10.1007/s00216-021-03279-7.

Acknowledgements This work has received funding from the Ministero dell'Università e Ricerca. PON Ricerca e Innovazione 2014-2020. Nanotecnologie chimiche green per la protezione sostenibile delle piante (NEMESI) ARS01 01002 CUP: F36C18000180005 and Regione Puglia. Design e sperimentazione di tecnologie innovative per la diagnosi precoce e trattamento del CoDiRO (DEMETRA) Cod. Y8V9H90.

Author contributions S. Di Masi: Data analysis, Writing - draft.

G. E. De Benedetto: Methodology, Data analysis, Writing - Reviewing and Editing.

C. Malitesta: Supervision, Writing - Reviewing.

M. Saponari: Data acquisition, Writing - draft.

C. Citti: Data acquisition.

G. Cannazza: Data acquisition, Writing - draft.

G. Ciccarella: Conceptualization, Project administration, Supervision, Writing - Reviewing.

Funding Open access funding provided by Università del Salento within the CRUI-CARE Agreement.

Data availability After acquisition, Agilent MassHunter data sets were converted into .mzML in centroid mode using MSConvert. mzMLdata files were uploaded to https://xcmsonline.scripps.edu. The datasets presented in this study can be found in online repositories. All results of the study are accessible in XCMS website with the following name and Job ID: name: final job ESI(+)MS1; JobID: 1424775 and name: final job ESI(-)MS1; JobID: 1424728.

\section{Declarations}

Conflict of interest The authors declare that they have no competing interests.

Open Access This article is licensed under a Creative Commons Attribution 4.0 International License, which permits use, sharing, adaptation, distribution and reproduction in any medium or format, as long as you give appropriate credit to the original author(s) and the source, provide a link to the Creative Commons licence, and indicate if changes were made. The images or other third party material in this article are included in the article's Creative Commons licence, unless indicated otherwise in a credit line to the material. If material is not included in the article's Creative Commons licence and your intended use is not permitted by statutory regulation or exceeds the permitted use, you will need to obtain permission directly from the copyright holder. To view a copy of this licence, visit http://creativecommons.org/licenses/by/4.0/.

\section{References}

1. Nigro F, Boscia D, Antelmi I, Ippolito A. Fungal species associated with a severe decline of olive in southern Italy. J Plant Pathol. 2013;95(3):668. 
2. Janse JD, Obradovic A. Xylella Fastidiosa: its biology, diagnosis, control and risks. J Plant Pathol. 2010;92:S35-48.

3. Martelli GP, Boscia D, Porcelli F, Saponari M. The olive quick decline syndrome in south-East Italy: a threatening phytosanitary emergency. Eur J Plant Pathol. 2016;144(2):235-43. https://doi. org/10.1007/s10658-015-0784-7.

4. Saponari M, Boscia D, Altamura G, Loconsole G, Zicca S, D'Attoma G, et al. Isolation and pathogenicity of Xylella fastidiosa associated to the olive quick decline syndrome in southern Italy. Sci. Rep. 2017;7(1):1-13.

5. Chatterjee S, Wistrom C, Lindow SE. A cell-cell signaling sensor is required for virulence and insect transmission of Xylella fastidiosa. PNAS. 2008;105(7):2670-5.

6. Bragard C, Dehnen-Schmutz K, Di Serio F, Gonthier P, Jacques MA, Miret JAJ et al. Update of the Scientific Opinion on the risks to plant health posed by Xylella fastidiosa in the EU territory. Efsa J. 2019;17(5): 05665 .

7. Djelouah K, Frasheri D, Valentini F, D'ONGHIA AM, Digiaro M. Direct tissue blot immunoassay for detection of Xylella fastidiosa in olive trees. Phytopathol Mediterr 2014:559-64.

8. Jorge TF, Rodrigues JA, Caldana C, Schmidt R, van Dongen JT, Thomas-Oates J, et al. Mass spectrometry-based plant metabolomics: metabolite responses to abiotic stress. Mass Spectrom Rev. 2016;35(5):620-49. https://doi.org/10.1002/mas.21449.

9. Peano C, Damiano F, Forcato M, Pietrelli A, Palumbo C, Corti G, et al. Comparative genomics revealed key molecular targets to rapidly convert a reference rifamycin-producing bacterial strain into an overproducer by genetic engineering. Metab. Eng. 2014;26:1-16.

10. Mentana A, Camele I, Mang SM, De Benedetto GE, Frisullo S, Centonze D. Volatolomics approach by HS-SPME-GC-MS and multivariate analysis to discriminate olive tree varieties infected by Xylella fastidiosa. Phytochem Anal. 2019;30(6):623-34.

11. Scala V, Pucci N, Salustri M, Modesti V, L'Aurora A, Scortichini $\mathrm{M}$, et al. Xylella fastidiosa subsp. pauca and olive produced lipids moderate the switch adhesive versus non-adhesive state and viceversa. PloS one. 2020;15(5):e0233013.

12. Colnaghi Simionato AV, da Silva DS, Lambais MR, Carrilho E. Characterization of a putative Xylella fastidiosa diffusible signal factor by HRGC-EI-MS. J Mass Spectrom. 2007;42(4):490-6. https://doi.org/10.1002/jms.1181.

13. Beaulieu ED, Ionescu M, Chatterjee S, Yokota K, Trauner D. Lindow S. Characterization of a Diffusible Signaling Factor from Xylella fastidiosa mBio. 2013;4(1):e00539-12. https://doi.org/10. 1128/mBio.00539-12.

14. Lindow S, Newman K, Chatterjee S, Baccari C, Lavarone AT, Ionescu M. Production of Xylella fastidiosa diffusible signal factor in transgenic grape causes pathogen confusion and reduction in severity of Pierce's disease. Mol Plant-Microbe Interact. 2014;27(3):244-54. https://doi.org/10.1094/mpmi-07-13-0197-fi.

15. Tautenhahn R, Patti GJ, Rinehart D, Siuzdak G. XCMS online: a web-based platform to process untargeted Metabolomic data. Anal Chem. 2012;84(11):5035-9. https://doi.org/10.1021/ac300698c.

16. Tsugawa H, Kind T, Nakabayashi R, Yukihira D, Tanaka W, Cajka $\mathrm{T}$, et al. Hydrogen rearrangement rules: computational MS/MS fragmentation and structure elucidation using MS-FINDER software. Anal. Chem. 2016;88(16):7946-58.

17. Worley B, Powers R. PCA as a practical indicator of OPLS-DA model reliability. Curr Metabolomics. 2016;4(2):97-103.

18. Yang B, Liu H, Yang J, Gupta VK, Jiang Y. New insights on bioactivities and biosynthesis of flavonoid glycosides. Trends Food Sci Technol. 2018;79:116-24.

19. Ognik K, Cholewińska E, Czech A. The effect of adding hesperidin, diosmin, quercetin and resveratrol extracts to feed for Turkey hens on selected immunological and biochemical blood indices. Ann Anim Sci. 2016;16(4):1101-14.

20. Bajraktari G, Weiss J. The aglycone diosmetin has the higher perpetrator drug-drug interaction potential compared to the parent flavone diosmin. J Funct Foods. 2020;67:103842.

21. Castejón Martínez ML, Montoya García T. Alarcón de la Lastra Romero C, Sánchez Hidalgo M. potential protective role exerted by Secoiridoids from Olea europaea L. in Cancer, cardiovascular, neurodegenerative, aging-related, and Immunoinflammatory diseases. Antioxidants. 2020;9(2):1-39.

22. Basile A, Giordano S, López-Sáez JA, Cobianchi RC. Antibacterial activity of pure flavonoids isolated from mosses. Phytochemistry. 1999;52(8):1479-82.

23. Yao M, Desilets H, Charles M, Boulanger R, Tweddell R. Effect of mycorrhization on the accumulation of rishitin and solavetivone in potato plantlets challenged with Rhizoctonia solani. Mycorrhiza. 2003;13(6):333-6.

24. Colinas M, Eisenhut M, Tohge T, Pesquera M, Fernie AR, Weber APM, et al. Balancing of B6 vitamers is essential for plant development and metabolism in Arabidopsis. Plant Cell. 2016;28:439 453. https://doi.org/10.1105/tpc.15.01033.

25. López-Carbonell M, Jáuregui O. A rapid method for analysis of abscisic acid (ABA) in crude extracts of water stressed Arabidopsis thaliana plants by liquid chromatography - mass spectrometry in tandem mode. Plant Physiol Biochem. 2005;43(4):40711.

26. Mauch-Mani B, Mauch F. The role of abscisic acid in plantpathogen interactions. Curr Opin Plant Biol. 2005;8(4):409-14. https://doi.org/10.1016/j.pbi.2005.05.015.

27. Ali M. Baek K-HJIJoMS. Jasmonic acid signaling pathway in response to abiotic stresses in plants. Int J Mol Sci. 2020;21(2):621.

28. Bouvier F, Isner J-C, Dogbo O, Camara B. Oxidative tailoring of carotenoids: a prospect towards novel functions in plants. Trends Plant Sci. 2005;10(4):187-94. https://doi.org/10.1016/j.tplants. 2005.02.007.

29. Ryan RP, An S-Q, Allan JH, McCarthy Y, Dow JM. The DSF family of cell-cell signals: an expanding class of bacterial virulence regulators. PLoS Pathog. 2015;11(7):e1004986.

30. Chatterjee S, Almeida RP, Lindow S. Living in two worlds: the plant and insect lifestyles of Xylella fastidiosa. Annu Rev Phytopathol 2008;46:243-71.

Publisher's note Springer Nature remains neutral with regard to jurisdictional claims in published maps and institutional affiliations. 